\title{
CENTRAL CORNEAL LIGHT SCATTER IN LONG-TERM DIABETICS
}

\author{
ROGER HOLDEN, G. ADRIAN SHUN-SHIN and NICHOLAS A. P. BROWN
}

\begin{abstract}
SUMMARY
Changes in corneal thickness have been correlated with severity of diabetic retinopathy. Measuring light scatter at the central cornea may reflect the changes in corneal thickness and hence the retinopathy. This study compared the peak central corneal light scatter between 22 insulin-dependent diabetic patients with disease of at least 10 years' duration and 29 controls. No significant difference was found in the light-scattering properties of the central cornea between the two groups. This technique does not offer an alternative method of screening for diabetic retinopathy.
\end{abstract}

Corneal involvement in diabetes has been demonstrated in clinical, morphological and biochemical studies. ${ }^{1-3}$ The light-scattering properties of the cornea are increased in corneal oedema. This is thought to be due either to disruption of the orientation of the collagen fibres or to local fluctuations in refractive indices of the oedematous cornea. Increased corneal thickness in diabetics with retinopathy has been demonstrated. ${ }^{4}$ This change is thought to be due to increased hydration. Any associated change in corneal light scattering may be related to the presence of retinopathy. Thus, measurement of corneal light scattering may offer a rapid, non-invasive method of screening for diabetic retinopathy. This study compared the light scattering, at the central cornea, of a group of long-term diabetics with that of a normal control group.

\section{PATIENTS AND METHODS}

Twenty-two insulin-dependent diabetics, with disease of at least 10 years' duration, were recruited consecutively from a diabetic eye clinic. Only patients with retinopathy of grades 2-4, using the modified Airlie House classification, ${ }^{5}$ were included. None had undergone laser photocoagulation within the previous 3 months. There was no other ocular pathology or history of contact lens wear. None were pregnant or taking the oral contraceptive pill. Twenty-nine normal controls were taken from a continu-

From: Clinical Cataract Research Unit, Nuffield Laboratory of Ophthalmology, Oxford, UK.

Correspondence to: Roger Holden, FRCS, FRCOphth, Department of Ophthalmology, Royal Liverpool University Hospital, Prescot Street, Liverpool L7 8XP, UK.

Eye (1994) 8, 44-45 C 1994 Royal College of Ophthalmologists ing trial investigating lenticular growth in adults. The same exclusion criteria applied. Age and sex matching was unnecessary as these factors do not affect central corneal light scatter. ${ }^{6}$ The right eye was used in all cases. Blood glucose and glycosylated haemoglobin assays were not performed as no correlation between these factors and corneal thickness has been demonstrated.

Anterior segment photography was performed on each patient using the Case 2000 CCD (charged couple device) Scheimpflug slit image camera. This equipment is an integral part of the Oxford Modular Cataract Image Analysis System (OMCIAS). ${ }^{8}$ The camera system ensures accurate fixation and alignment of the eye, allowing image grabbing only when the eye is in the correct position. The image, grabbed onto a framestore and displayed on a monitor, is stored digitally. Each image is standardised by reference to two standard neutral density filters. Once calibrated, the image analysis software prepares a microdensitometry trace by averaging the values of an axial horizontal strip 10 pixels in width. A typical trace from an image displaying the scatter throughout the central cornea and lens is shown in Fig. 1. Light scatter is measured in arbitrary neutral density units (ANDU) as the calibration system is not directly equivalent to SI notation.

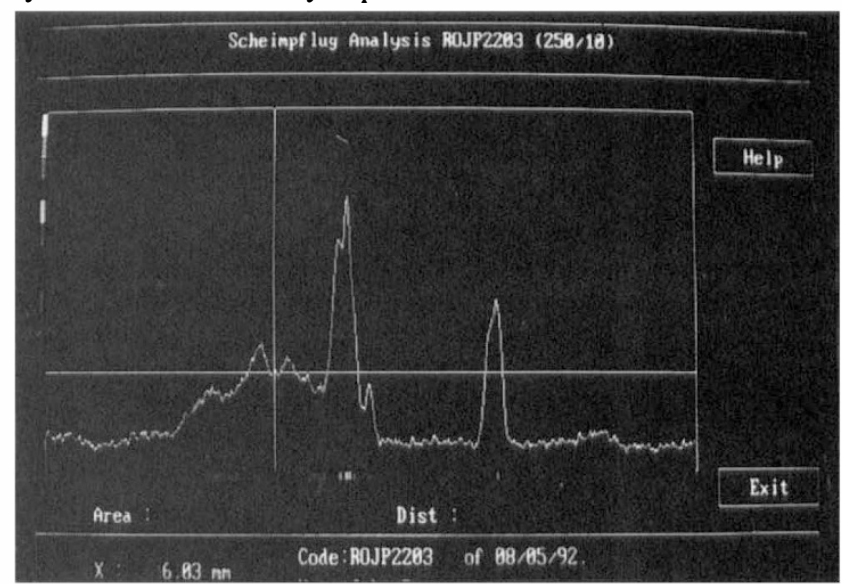

Fig. 1. Typical microdensitometry tracing of anterior segment. The cross hairs are aligned at the centre of the lens. The first tracing to the right of the picture is that of the central corneal light scatter. 
Table I. Mean age and peak central corneal light scatter in the diabetic and control groups

\begin{tabular}{lllll}
\hline & Diabetics & Controls & $p$ & $95 \%$ CI \\
\hline Age $(\mathrm{yr})$ & $54.5(13)$ & $63.5(11)$ & $0.02^{*}$ & $-16 \%$ to $1.5 \%$ \\
Corneal light scatter (ANDU) & $21.8(2.6)$ & $22.5(1.5)$ & 0.22 & $-20 \%$ to $5 \%$ \\
\hline
\end{tabular}

Values are the mean $(\mathrm{SD})$.

ANDU, arbitrary neutral density units; $95 \% \mathrm{CI}, 95 \%$ confidence interval.

*Significant at the $5 \%$ level.

For the central corneal portion of the image, three measurement parameters are possible. These are biometry (corneal thickness), peak scatter and planimetry (area under the scatter curve). In this study peak corneal scatter was measured, as the reliability of the other two measurements on this system is relatively low. Separate scattering peaks noted at higher magnifications by Smith et al. ${ }^{6}$ are not defined on the Case 2000 system. All photography and image analysis was performed by the same investigator (R.H.), who was masked as to the grouping of each patient. The repeatability of the peak corneal scatter was assessed for the purposes of the study. Twenty-two of the control group were photographed on two separate days and the peak corneal scatter measured in a masked fashion.

The mean peak corneal scatter was compared between the two groups using Student's two-tailed $t$-test. The repeatability was calculated using the coefficient of variation.

\section{RESULTS}

The coefficient of variation, as a measure of intra-observer variation of photography and analysis combined, was 0.99 $(y=0.15+0.99 x)$. Mean age and peak corneal scatter are shown in Table I. Mean duration of diabetes in the study group was 24.5 years (standard deviation 10.4 years). No significant difference in peak central corneal scatter was demonstrated between the two groups.

\section{DISCUSSION}

Measurement of the peak corneal light scattering using the OMCIAS did not demonstrate any significant difference between a group of long-term diabetics and normal controls. The relatively small numbers in each group are reflected in the wide confidence intervals. The excellent repeatability of the system for measuring corneal scatter is comparable with that for lenticular scatter measurement. ${ }^{9}$

Duration of diabetes has generally been taken as the best predictor of retinopathy. It was with this in mind that we chose to recruit long-term diabetics. Stolwijk et al. ${ }^{10}$ found that corneal autofluorescence correlated more strongly with grade of retinopathy than with duration of diabetes. This change in fluorescence was postulated to be due to both accumulation of fluorophores and changes in mitochondrial flavoprotein. Whether these changes would alter corneal light scatter, perhaps by increasing absorption and negating any increased scatter due to hydration, is open to conjecture.

Changes in corneal hydration and thickness, the most likely source of increased scatter, have been demonstrated in human and animal studies. Herse ${ }^{11}$ found that in the uncontrolled diabetic rabbit the corneal stroma underwent the least change in hydration in relative terms. Peak light scattering occurs in the anterior stroma; therefore measurement of this feature may not be the ideal way of detecting overall changes in corneal hydration. However, because the stroma constitutes the vast majority of corneal thickness, it is reasonable to expect that any significant change in scatter due to hydration would be detected using this equipment. Also, the amount of scatter from the epithelium and endothelium is relatively small. Any increase due to hydration of these layers would be unlikely to register a significant change in the overall scattering of light. Planimetric measurement (the area under the entire scatter curve) may be superior theoretically, but this advantage is outweighed by the inferior repeatability of the method.

In summary we did not detect any difference in central corneal light scatter in a group of insulin-dependent diabetic patients compared with a normal control group. The potential for this method as a screening tool for retinopathy is limited.

Key words: Cornea, Diabetic retinopathy, Light scatter.

\section{REFERENCES}

1. Herse PR. A review of the manifestations of diabetes mellitus in the anterior eye and cornea. Am J Optom Physiol Opt 1988;65:224-30.

2. Marano CW, Matschinskey FM. Biochemical manifestations of diabetes mellitus in microscopic layers of the cornea and retina. Diabetes Metab Rev 1989;51:1-15.

3. Gobbels M, Spitznas M, Oldendoerp J. Impairment of corneal epithelial barrier function in diabetes. Graefes Arch Clin Exp Ophthalmol 1989;227:142-4.

4. Busted N, Olsen T, Schmitz O. Clinical observations on the corneal thickness and corneal endothelium in diabetes mellitus. Br J Ophthalmol 1981;65:687-90.

5. The Diabetic Retinopathy Study Research Group. Report 7: A modification of the Airlie House classification of diabetic retinopathy. Invest Ophthalmol Vis Sci 1981;21:210.

6. Smith GTH, Brown NAP, Shun-Shin GA. Light scatter from the human central cornea. Eye 1990;4:584-8.

7. Pierro L, Brancato R, Zaganelli E. Correlation of corneal thickness with blood glucose control in diabetes mellitus. $\mathrm{Br}$ J Ophthalmol 1981;65:687-90.

8. Sparrow JM, Brown NAP, Shun-Shin GA, Bron AJ. The Oxford Modular Cataract Image Analysis System. Eye 1990;4:638-48.

9. Harris ML, Smith GTH, Brown NAP. Inter- and intra-observer reproducibility of the new Oxford CCD Scheimpflug camera. Eye 1991;5:487-90.

10. Stolwijk TR, van Best JA, Oosterhuis JA, Swart W. Corneal autofluorescence: an indicator of diabetic retinopathy. Invest Ophthalmol Vis Sci 1992;33:92-7.

11. Herse PR. Corneal hydration control in normal and alloxaninduced diabetic rabbits. Invest Ophthalmol Vis Sci 1990; 31:2205-13. 